mother-infant interactions, however, indicates that these are not asymmetrical and may have been a focus of intense natural selection.

The discussions in The Origins of Meaning of ritualization, emulation, imitation, learning and 'machiavellian intelligence' are insightful. So is the review of communications that include gaze-following, attention-seeking behaviour, pointing, begging and alarm calls in nonhuman primates and/or young humans, and their possible implications for the evolution of referential language. After considering niche construction, kin and sexual selection, and reciprocal altruism, Hurford concludes that communicative cooperation and trust may have been the most crucial factors in the emergence of language.

There are some titillating nuggets in this book, such as a discussion of how the FOXP2 gene was mistakenly accepted as the 'magic bullet' responsible for language evolution. Even better is the extent to which academics from different countries use language competitively to show off - guess where Americans rank?

Has Hurford achieved his goal of describing the evolutionary foundations of language? Yes, elegantly and in accomplished detail that should be accessible to all those specialists the book targets. I look forward to finding out what he thinks happened next in the second volume.

Dean Falk is in the Department of Anthropology, Florida State University, Tallahassee, Florida 32306-7772, USA. She is the author of Braindance: New Discoveries about Human Origins and Brain Evolution.

\title{
Space and time gift-wrapped
}

\author{
Very Special Relativity: An illustrated \\ Guide \\ by Sander Bais \\ Harvard University Press: 2007. 144 pp. \\ $\$ 20.95, \notin 13.95$
}

\section{Andrew Jaffe}

Sander Bais's Very Special Relativity is a brief overview of Albert Einstein's 1905 theory of space and time, an esoteric topic that one would presume to be directed at a narrow audience. But this book would not be out of place on a coffee table, with its handsome design, thick, coloured papers and imaginative graphics that match the high standard of its content. We expect art books, not science texts, on our coffee tables, assuming that in science it is the ideas that matter, not the visual presentation.

Here, the elegant illustrations help Bais lead the reader from Einstein's postulates through the ideas of simultaneity, inertial frames, time dilation and relativistic energy and momentum, eschewing the usual admonitions against equations. The author's clever idea of pairing every page of text with a space-time diagram (a graphical tool actually used by relativists) to illustrate the concepts and mathematics suits the geometrical basis of its subject perfectly. It allows Bais to stop just short of using calculus - although the definitions of things such as 'slope' and 'tangent' are taken for granted, and he does allow a $\mathrm{d} p / \mathrm{d} t$ to slip in when discussing momentum and forces. He manages to navigate all the way to $E=m c^{2}$ and to give us a taste of Einstein's even more miraculous work of the following decade, his general relativity theory of gravitation.

Very Special Relativity is aimed at an underserved market: keen high-school students will welcome it because it extends beyond qualitative discussion of 'modern' physics and popular-science books. With its brainteaser problems, it should also work as an undergraduate introductory textbook. Readers who haven't exercised their mathematical and geometric muscle since they were in school might find it tough going.

It is rare for science books to rate as objects in their own right, but Very Special Relativity is a lovely little object. You could easily imagine a web-based version of it, with a bit of animation to serve its pedagogical needs. Still, there is some quality about the hard covers and high resolution that even my 26 -inch screen wouldn't be able to capture. No longer is there an excuse for physics textbooks to be expensive, boring, thick or stuffed with equations in order to qualify as good teaching material. Andrew Jaffe is professor of astrophysics and cosmology in the Astrophysics Group, Blackett Laboratory, Imperial College London, London SW7 2AZ, UK.

\section{PAINTING}

\section{Hidden depths spotted}

Emiliano Feresin

Nuclear physicists have revealed that the work of Sicilian artist Antonello da Messina (1430-79) may have been ahead of its time. He was among the first, they suggest, to subtly refine the shading in his paintings by spotting them with lacquer. When applied to particular areas of a painting, this glaze, made from red pigment mixed with oil, enhances the impression of depth.

Pier Andrea Mandò and his colleagues at the Italian National Institute of Nuclear Physics' Labec laboratory in Florence took advantage of the availability of Antonello's Portrait of a Man (1476) during its restoration this summer. They had already developed and used non-destructive PIXE (for 'particleinduced X-ray emission') scans to reconstruct the spatial distribution and concentration of chemical elements on the surface of some types of artistic work, such as gold laces and embroideries. They wanted to try the technique for the first time on a painting.

The physicists identified traces of aluminium and iron - a typical signature of lacquers in tiny, uneven spots over the red cloak of the unknown subject. Although such glazes were already in use in the fifteenth century, they were normally applied with a brush over an entire surface. The scan reveals a weave-like imprint across the red cloak, suggesting to the restorers that here the shiny substance was dabbed on with a cloth.

Later, similar effects were created by great masters such as Leonardo da Vinci (14521519), who used his finger, and Rembrandt (1606-69), who used the end of his brush. But if Mandò and his colleagues are correct, it was Antonello - best known for introducing certain techniques of Flemish masters into Italy - who pioneered the effect. Emiliano Feresin is an intern in Nature's Munich office.

The restored Portrait of a Man, pictured here, can be viewed at the Civic Museum of Ancient Art in Palazzo Madama, Turin: www.palazzomadamatorino.it.

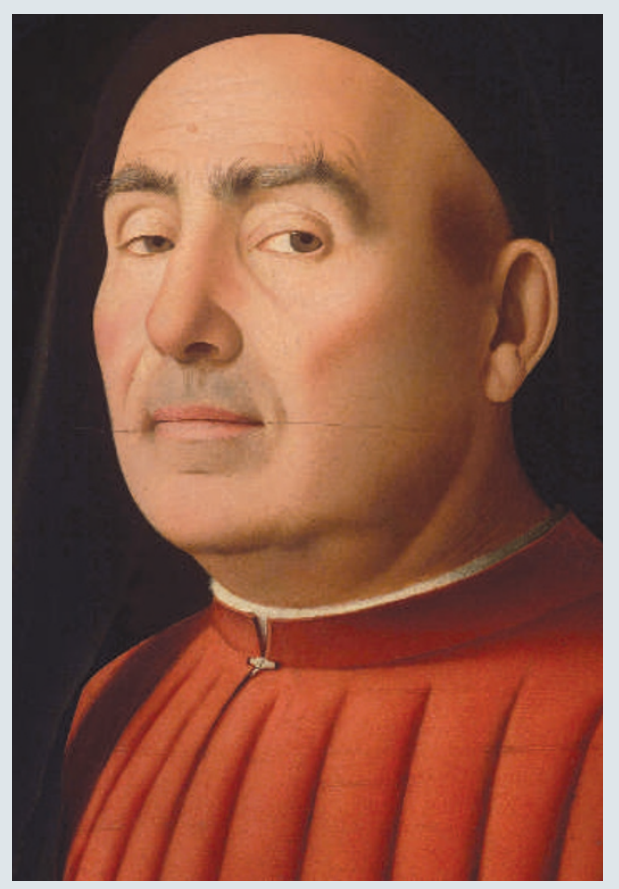

